descriptions of all genera and species are given, together with brief notes on habitat and distribution. Most species are illustrated by clear line diagrams.

This book represents years of painstaking and accurate work on this difficult group of fungi. In spite of the delay in its publication, it is a substantial and welcome addition to the literature and will prove to be invaluable, not only to Indian mycologists, but to all students of the Hyphomycetes and to plant pathologists.

The book is worthy of stronger binding (which does not stand up to normal handling of a volume of more than 900 pages) and of better quality paper. It is to be hoped that its success will warrant an early revision giving full justice to the author's scholarship and critical powers. L. E. HAWKER

\section{Membranes and Ions}

Biophysics and Physiology of Excitable Membranes. Edited by William J. Adelman, jun. Pp. xiv +527 . (Van Nostrand Reinhold: New York and London, January 1972.) £12.25.

Membranes. Edited by George Eisenman. Vol, 1. Macroscopic Systems and Models. Pp. xix +333. (Marcel Dekker: New York, May 1972.) \$19.50.

IT is curious that at a time when research on cell membranes has become such a fashionable field, there should be so few textbooks dealing adequately with certain aspects of the subject. The appearance of a book from which, to quote its dust cover, one "can gain a broad view of the theoretical approaches and technical procedures now used in excitable membrane biophysics" is therefore welcome. But as is too often the case with multi-author texts, the quality of the articles is variable and there are some surprising gaps in their coverage. Thus as far as voltage clamp studies are concerned, it seems a pity that a critical and helpful account of the application of the technique to myelinated fibres should be accompanied by one on giant axons that is marred by its cursory dismissal of the problems arising from the potential drop across the series resistance $R_{\mathrm{s}}$ due to the presence of the Schwann cell. As K. S. Cole points out in his historical survey, Hodgkin and Huxley took some pains to compensate electronically for at least part of $R_{s}$, but many of their successors have simply ignored the series resistance. In a fresh axon the resulting current-voltage curves may be displaced by many millivolts from their correct position. The subject is not well served by glancing over this admittedly technically troublesome point when at the same time consider- able space is devoted to the questionable advantages of voltage-clamping with continuously varying command potentials instead of discrete steps.

It is surely odd that a chapter entitled "The Thermodynamic Foundations of Membrane Physiology" should make no mention at all of experimental investigations on the calorimetry of the nerve impulse. And although the mechanism of active transport of ions in nerve and muscle is dealt with very adequately, almost nothing is said about the measurement of the downhill ionic movements during nervous activity. There are useful chapters on other topics such as the X-ray analysis of myelin structure, optical studies, perfusion of giant axons, drug action, and experiments on model membranes. But the claim of the book to conduct a thorough "in-depth" examination does not seem entirely justified.

A similar claim is made for the volume edited by Professor Eisenman, this time more justifiably since it is the first of a series of unspecified length. Volume 1 sets the stage, containing accounts of the physical chemistry and theoretical background for the penetration of ions across different types of membrane, such as those with neutral pores, ion-exchange resin membranes, liquid membranes and dry silicate membranes. Volume 2 will be wholly concerned with the ionic conductance of artificial lipid bilayers incorporating carrier-type and tunnel-forming ionophores. This seems a logical scheme, and the last two chapters in Volume 1 are therefore a little out of place, consisting as they do of some purely theoretical arguments about excitable membranes, and of a mainly technical account of a new method for turning squid axons inside-out. In spite of the ingenuity of this procedure it is not clear that it has any marked advantage over existing perfusion techniques.

\section{R. D. KEYNES}

\section{Human Error}

Human Factors in Highway Traffic Safety Research. Edited by T. W. Forbes. Pp. xix +419 . (Wiley: New York and London, June 1972.) £7.85.

ScIENTIFIC investigations towards an understanding of driving behaviour and a theory of accident causation have been conducted in such diverse fields as highway and traffic engineering, ergonomics, psychology, and driver education. Perhaps because of this, such research is not always accessible to the engineers who are charged with the task of designing a safe transport system for human use. Human factors is one of the newer engineering disciplines which attempts to assess the design implications of behavioural studies.

This book, which has contributions from seventeen authors, specialists in a particular area of human factors studies, summarizes research on the biographical and physiological characteristics of drivers, factors in sign legibility, other methods of presenting information to drivers, skills, judgment and information acquisition in controlling vehicles in traffic, impairment of driver performance due to such factors as fatigue, alcohol and drugs, the effect of psycho-social factors such as stress, driver education and improvement. It can be seen from the comprehensive range of topics covered that of necessity it can only serve as an introduction. Each section is extensively referenced, however, thereby making a very valuable contribution. Consequently this may be of interest to students on courses of highway and traffic engineering as well as practitioners in the field of road safety. The extent to which it can be effective in arousing students' interest in the implications of their work as engineers, as an isolated course, is unknown.

The main area of concern, however, is the extent to which an approach such as this can achieve the stated objective of designing the man-machine environment task and/or system to fit human capabilities wherever possible. In principle this seems to be a very interesting approach. But most of the analysis is conducted in a vacuum quite distinct from actual accident occurrence. No attempts were made to show how this type of formal model, especially with regard to car following, overtaking, and so on, had any predictive validity. Indeed, the author concluded that drivers do a remarkably good job even in a system where breakdown appears to be inevitable. Perhaps this very observation suggests that the system analysis approach is not valid.

The cybernetic models proposed in most chapters to describe the drivercar-road environment system equated man with a limited capacity information-processing channel and driving as a negative feedback control system. It seems unlikely that a theory of driving which makes such simplistic assumptions about the driver will be very useful. Such a theory denies the possibility that he may initiate any action. Driving is only one of a range of socia! activities which is influenced by the behaviour of other people and as such is affected by the prevailing social mores which may be antipathetic to safety. While this book gives a good coverage of topics related to human factors, contributions from social psychologists are omitted.

Given this uncertainty about the validity of the model, the ability of this 